\title{
The Aesthetics and Fractal Dimension of Electric Sheep
}

\author{
SCOTT DRAVES \\ RALPH ABRAHAM* \\ PABLO VIOTTI** \\ FREDERICK DAVID ABRAHAM*** \\ JULIAN CLINTON SPROTT****
}

December 24, 2006

\footnotetext{
Spotworks, 2261 Market St \#158, San Francisco, CA 94114 USA

* Mathematics Dept., University of California, Santa Cruz, CA 95064 USA

** Politics Dept., University of California, Santa Cruz, CA 95064 USA

*** Blueberry Brain Institute, 1396 Gregg Hill Road, Waterbury Center, VT 05677 USA

**** Physics Dept., University of Wisconsin, Madison, WI 53706-1390 USA

Running Head: Aesthetics and Fractal Dimension
} 


\begin{abstract}
Physicist Clint Sprott demonstrated a relationship between aesthetic judgments of fractal images and their fractal dimensions [1993]. Scott Draves, aka Spot, a computer scientist and artist, has created a space of images called fractal flames, based on attractors of twodimensional iterated function systems. A large community of users run software that automatically downloads animated fractal flames, known as 'sheep', and displays them as their screen-saver. The users may vote electronically for the sheep they like while the screen-saver is running. In this report we proceed from Sprott to Spot. The data show an inverted U-shaped curve in the relationship between aesthetic judgments of flames and their fractal dimension, confirming and clarifying earlier reports.
\end{abstract}




\section{Introduction}

This is a report on a new study of aesthetic judgments made by a large community participating over the internet in a collective art project, the Electric Sheep, created by Scott Draves. To this system we have applied the ideas of Clint Sprott of fractal dimension as an aesthetic measure. Our study thus combines the Electric Sheep of Draves and the fractal aesthetics of Sprott.

The Electric Sheep home page is available from electricsheep.org. We begin by describing the Electric Sheep network, and then our project and results. In short, we find the aesthetic judgments of an internet community of about 20,000 people on a set of 6,400 fractal images confirms the earlier findings of a unimodal distribution with a peak near dimension 1.5. We then review the history of fractal aesthetics to put this work in context, and conclude.

\section{The Electric Sheep Network}

Fractal Flames [Draves, 2004] are a generalized and refined kind of iterated function system, some examples appear in Figs. 1 and 2. They and the Electric Sheep network change over time as new versions are released. Here we describe them as they were when the data for this paper were collected. At that time in 2004, a flame consisted of two to six nonlinear mappings in two dimensions. Each of the nonlinear mappings consists of an affine 2x3 matrix 
composed with a dot product of a parameter vector and a collection of about 20 hand-designed nonlinear basis functions, making for a total parameter space of about 160 floating point numbers. A point in this space is called a genome.

Whereas traditional iterated function systems are binary images where each pixel has either been plotted or not, fractal flames are full-color images with brightness and color. The brightness is determined by a tone map based on the logarithm of the density of the attractor, or number of particles, at that pixel. The color is determined by adding a 3rd coordinate to the iteration and looking it up in a palette.

The animation of a sheep comes from rotating the matrix parts of its genome, hence the animation loops seamlessly. Sheep are 128 frames long, hence lasting 4-5 seconds during playback.

The Electric Sheep [Draves, 2005] consists of the sheep server and a large number of clients, which are screen-savers on internet-connected computers owned by users. When they run, the clients connect to the server to form a distributed super-computer, which we call the render farm, an idea pioneered by SETI@Home [Anderson, 2002].

The server keeps about 40 sheep alive, replacing old sheep with new ones every fifteen minutes or so, as they are completed by the render farm. The sheep are downloaded to the user's client. The client may hold thousands of sheep taking gigabytes of disk space, but the default is only enough space for 100 sheep. If the client's buffer is full, its oldest and lowest rated sheep are 
deleted to make room for the new.

Users see the sheep displayed by their screen-savers, and may vote for or against a sheep by pressing the up and down arrow keys. The votes are tallied by the server into a rating for each sheep.

Genomes for new sheep come from three sources: randomness, a genetic algorithm, and user contributions:

random These genomes have most matrix coefficients filled in with random numbers from $[-1,1]$, or to a simple symmetry transformation (for example, rotation by 60 degrees). In each mapping, one nonlinear coefficient is set to one and the rest to zero.

evolved These were produced by a genetic algorithm with mutation and cross-over operators. A sheep's chance for reproduction is proportional to its rating so the most popular sheep reproduce the most. Mutations come from adding noise to the parameters in the genome. Cross-over is done by combining parts of the genomes of two sheep to form the child genome. See Draves [2005] for a detailed explanation.

designed These were contributed by users of Apophysis, a Microsoft Windows GUI-application for designing fractal flames by manipulating the parameters in the genome in real-time at draft quality. The matrices are represented by dragable triangles, and the nonlinear coefficients with ordinary text widgets. 
All sheep, from server reset to reset, comprise a flock. In this project, we have used the database of flock 165, which lived from March through October of 2004. The server maintains records of all sheep of the flock, along with their peak ratings, that is, the highest rating attained during the sheep's lifetime, hereafter simply refered to as the rating. These databases are available for download from the sheep server.

\section{The Project and Findings}

In the spirit of experimental aesthetics pioneered by Clint Sprott, we expected a correlation between the fractal dimension and the rating of the sheep. Fractal flames are attractors, or fixed points, of two-dimensional functions, with an independent third dimension displayed via a color palette, and brightness determined by density. For simplicity we ignored the color so the dimension computed here is a real number between zero and two.

Each frame of a sheep animation has a Fractal Dimension, FD. This is the correlation dimension, or D2 of Grassberger and Procaccia, which we computed by the algorithm of Sprott [1983]. This works by measuring correlations between points produced by the iteration, rather than by analyzing the resulting image.

The FD of a sheep varies over time, so we define the Average Fractal Dimension or AFD of a sheep to be the average of 20 frames evenly spaced (by rotations of 18 degrees) throughout the sheep. 
Unfortunately it would take too long to compute the AFD of all the sheep, so Fig. 3 uses the FD of the first frame of each sheep. Fortunately FD and AFD differ little: Fig. 4 shows the similarity between FD and AFD. We computed AFD for the 1109 sheep with non-zero rating. Figure 5 shows a scatter plot of AFD vs FD, the correlation is 0.92 .

The flock 165 database contained records of 6,396 sheep where we could compute the dimension: 2,604 from the genetic algorithm, 2,598 random, and 1,194 user-designed. We plot two frequency distributions with these four categories: on the top in Fig. 3 is the number of sheep of that dimension (bins are 0.05 wide), on the bottom is the sum total of ratings of sheep of that dimension.

In short, we find that sheep of AFD between 1.5 and 1.8 were greatly favored by users. The average FD of the designed sheep was 1.49 and the average AFD of all the sheep weighted by rating was 1.53.

Does this distribution result from user preference and evolution, or simply a quirk of the algorithm that produces the random genomes? Because the distribution of purely random genomes in the top of Fig. 3 is markedly different (with a peak at the maximum possible of 2), but the distribution of hand-designed sheep is very similar, we determine the bias results from human preference.

Or perhaps the distribution results from the distribution of the sheep, rather than a distribution of preference. For example, if users voted for sheep randomly, but more sheep of dimension 1.5 were produced, we would 
also see a peak at 1.5. To account for this we computed the average rating of sheep of each dimension (again the bins are 0.05 wide). The results appear in Fig. 6. The peak moves from 1.5 to between 1.6 and 1.7. However there is also a peak at 1.15. It is unknown if this is an anomaly due to the low sample size on this end of the graph, or if it represents a consistent aesthetic preference.

\section{Fractal Aesthetics}

Experimental aesthetics has a long history. For example, Galileo's father performed experiments on the aesthetics of musical intervals according to different musical scales, or tunings, published in 1588. Gustav Fechner founded the field in name starting with his investigation of the golden rectangle [1876]. In 1933, George David Birkhoff, one of the first American mathematicians of note, suggested a formula for the complexity of an image, and proposed it as an aesthetic measure. And in 1938, Rashevsky, the father of mathematical biology, suggested a connection between aesthetics and neurophysiology (see Berlyne [1971]). Mandelbrot's work also brought attention to the relationship of fractal mathematics and dynamical systems to the field of aesthetics [Mandelbrot, 1983; Peitgen \& Richter, 1996].

Our own basic area of fractal aesthetics began with the work of Clint Sprott [1993a,b; 1994; 2003]. This work proposed fractal dimension as a measure of complexity of a fractal image, and examined its relationship to 
aesthetic perception.

The 1994 paper reports a preference peak at dimension $1.51 \pm 0.43$ for $2 \mathrm{D}$ iterated function systems by averaging the dimension of the 76 images rated 5 on a scale of 1 to 5 by Sprott himself. In our experiment, the average AFD of the 76 highest-rated sheep (with ratings of 25 to 170 ) was $1.52 \pm 0.23$, a remarkable agreement.

Sprott's book [1993] reports a preferred dimension of $1.30 \pm 0.20$ for strange attractors. This work was extended by Aks and Sprott [1996], who measured aesthetic judgments of 24 subjects to 324 fractal images, and by Fred Abraham et al. [2001] and Mitina \& Abraham [2003], who measured the responses of 18 subjects to 16 images and found dimension 1.54 was preferred over $0.59,1.07$, and 2.27 .

In contrast to the Electric Sheep and this work, Mitina and Abraham [2003] used images created as chaotic attractors of a single iterated polynomial function in three dimensions, with the third dimension shown as a color. Their correlation dimensions were computed from three-dimensional data, and thus vary between zero and three.

\section{Conclusions}

We have confirmed the findings of Sprott, Aks and Sprott, and Fred Abraham et al. Our group of experimental subjects, as well as the number of images used, is much larger than the earlier studies, however Fig. 6 remains to be 
explained. In addition, our research opportunity, the Electric Sheep project, is ongoing, evolving in complexity, and increasing in size. We have thus the opportunity to continue posing hypotheses and seeking new results. 


\section{References}

[1] Abraham, F.D., Sprott, J.C., Mitina, O., Osorio, M., Dequito, E.A., Pineli, A.M. [2001 and In preparation] Judgments of time, aesthetics, and complexity as a function of the fractal dimension of images formed by chaotic attractors. Presented at the annual conference of the Society for the Chaos Theory in Psychology and the Life Sciences.

[2] Aks, D.J., \& Sprott, J.C. [1996] "Quantifying aesthetic preference for chaotic patterns," Empirical Studies of the Arts, 14(1), 1-16.

[3] David Anderson et al [2002] "SETI@home: An experiment in publicresource computing," Communications of the ACM 45: 56-61.

[4] Avital, T., \& Cupchik, G.C. [1998] "Perceiving hierarchical structure in nonrepresentational paintings," Empirical Studies of the Arts 16(1), $59-70$.

[5] Berlyne, D.E. [1971] Aesthetics and Psychobiology (Appleton-CenturyCrofts, New York).

[6] Berlyne, D.E., \& Olgivie, J. [1974] "Dimensions of perception of paintings." In D.E. Berlyne (Ed.), Studies in the New Experimental Aesthetics: Steps toward an Objective Psychology of Aesthetic Appreciation (Hemisphere, Washington, DC).

[7] Birkhoff, G.D. [1933] Aesthetic Measure (Harvard, Cambridge, MA). 
[8] Davis, R.C. [1936] "An evaluation and test of Birkhoff's aesthetic measure and formula," J. General Psychology, 15, 231-240.

[9] Draves, Scott [2005] "The Electric Sheep Screen-Saver: A case study In aesthetic evolution," Applications of Evolutionary Computing LNCS, 3449.

[10] Draves, Scott. [2004] "The fractal flame algorithm," available from http://flam3.com/flame.pdf.

[11] Fechner, G.T. [1876] Vorschule der Äesthetik (Breitkopf \& Härtel, Leipzig).

[12] Grassberger, P., \& Procaccia, I. [1983] "Measuring the strangeness of strange attractors," Physica D, 9, 189-208.

[13] Kuhl, J. [1986] "Motivational chaos: a simple model." In D.R. Brown \& J. Veroff (Eds.), Frontiers of Motivational Psychology (Springer-Verlag, Berlin).

[14] Mandelbrot, B.D. [1983]. The Fractal Geometry of Nature (Freeman, New York).

[15] Mitina, O.V., \& Abraham, F.D. [2003]. "The use of fractals for the study of the psychology of perception: psychophysics and personality factors, a brief report," Int. J. Modern Physics C, 14(8), 1-14. 
[16] Peckham, M. [1965]. Man's Rage for Chaos: Biology, Behavior and the Arts (Chilton, New York).

[17] Peitgen, H.-O., \& Richter, P.H. [1996]. The Beauty of Fractals: Images of Complex Dynamical Systems (Springer, Berlin).

[18] Pickover, C.A. [1990]. Computers, Pattern, Chaos, and Beauty (St. Martins, New York).

[19] Rashevsky, N. [1938]. "Contribution to the mathematical biophysics of visual perception with special reference to the theory of aesthetic values of geometrical patterns," Psychometrika, 3, 253-271.

[20] Sprott, J.C. [1993a]. "Automatic generation of strange attractors," Computers 83 Graphics 17, 325-332.

[21] Sprott, J.C. [1993b]. Strange Attractors: Creating Patterns in Chaos (M\&T, New York).

[22] Sprott, J.C. [1994]. "Automatic generation of iterated function systems," Computers and Graphics, 18, 417-425.

[23] Sprott, J.C. [2003]. Chaos and Time-series Analysis (Oxford: Oxford).

[24] Taylor, R.P., Micolich, A., \& Jonas, D. [1999]. "Fractal analysis of Pollock's drip paintings," Nature, 399, 422.

[25] Taylor, R.P., Spehar, B., Wise, J.A., Clifford, C.W.G., Newell, B.R., \& Martin, T.P. [2003] "Perceptual and physiological responses to 
the visual complexity of Pollock's dripped fractal patterns," Nonlinear Dynamics, Psychology, and Life Sciences, 9(1), 89-114. Also at: http://materialsscience.uroregon.edu/taylor/art/Boston.pdf.

[26] Wundt, W.M. [1874]). Grundgzüge der Physiologischen Psychologie (Engelmann , Leipzig). 


\section{Figure Captions}

Figure 1. Two sheep (fractal flames) chosen by Draves from the screensaver according to his own aesthetic.

Figure 2. Twelve example sheep. The fractal dimension increases left to right from 1.25 to 1.5 to 1.7 to 2.0 , and the aesthetic rating increases top to bottom from 5 to 10 to 20 .

Figure 3. The top graph shows the frequency distributions of the number of sheep (on the vertical axis) against their fractal dimension (on the horizontal). The bottom graph shows the sum of the ratings of sheep vs. fractal dimension (FD). The lines are for the three categories of sheep: designed by users, random de novo, and evolved, i.e. from the genetic algorithm, plus one line for all the sheep combined.

Figure 4. Comparison of Fractal Dimension (FD), sampled at time 0, and Average Fractal Dimension (AFD), computed from 20 evenly spaced samples. These curves are for all sheep combined.

Figure 5. Scatter plot of Fractal Dimension (FD) on the horiziontal axis vs Average Fractal Dimension (AFD) on the vertical, 1109 samples. The correlation is 0.92 . 
Figure 6. Graph of average rating (left vertical axis) and the sample size (right vertical axis) against fractal dimension (FD) on the horizontal axis. The ratings line is omitted where it has less than 100 samples. 

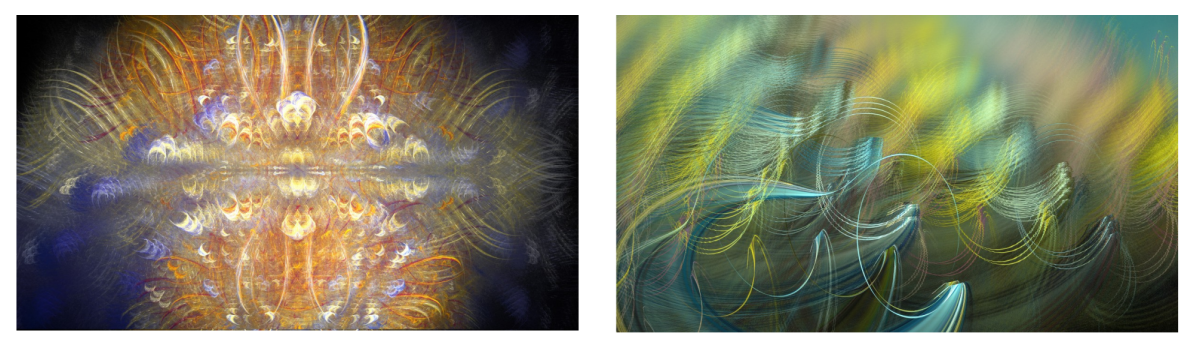

Figure 1:

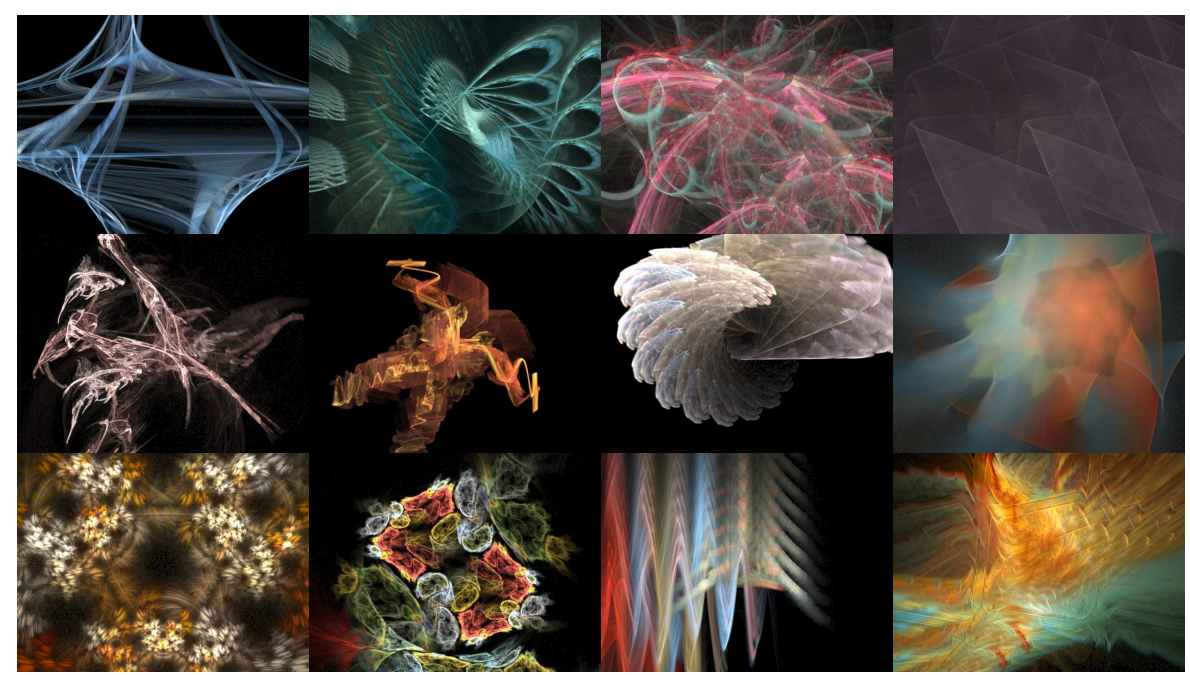

Figure 2: 

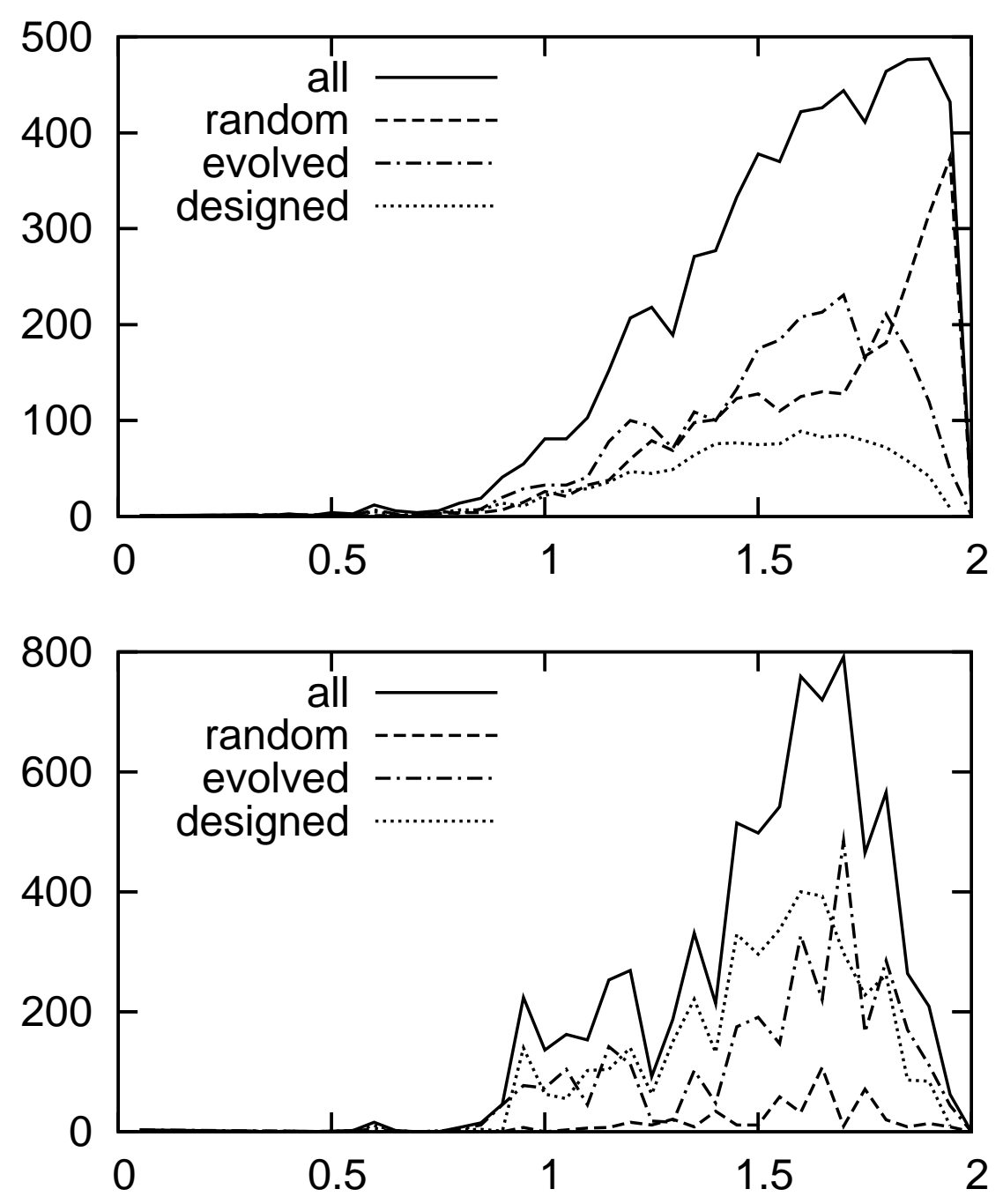

Figure 3: 


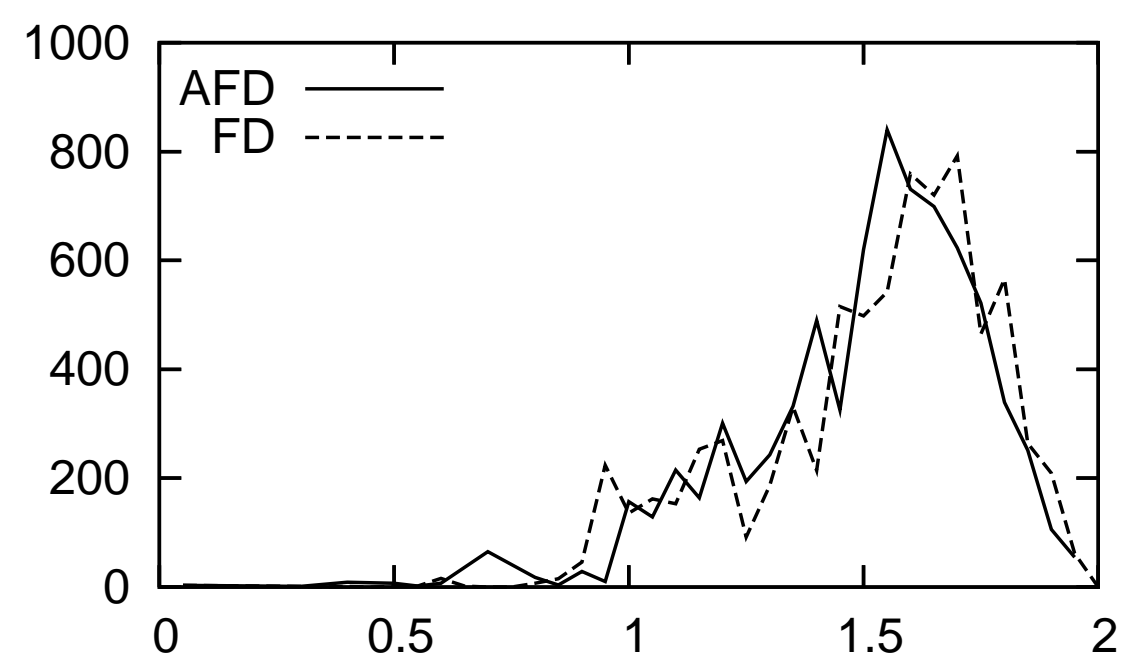

Figure 4:

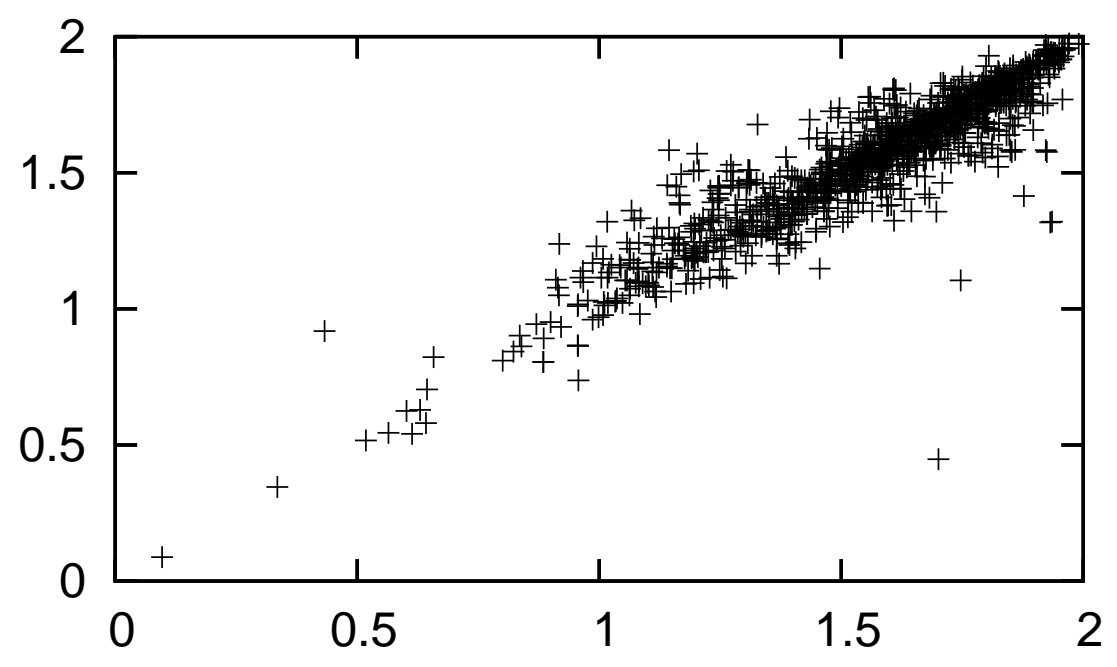

Figure 5: 


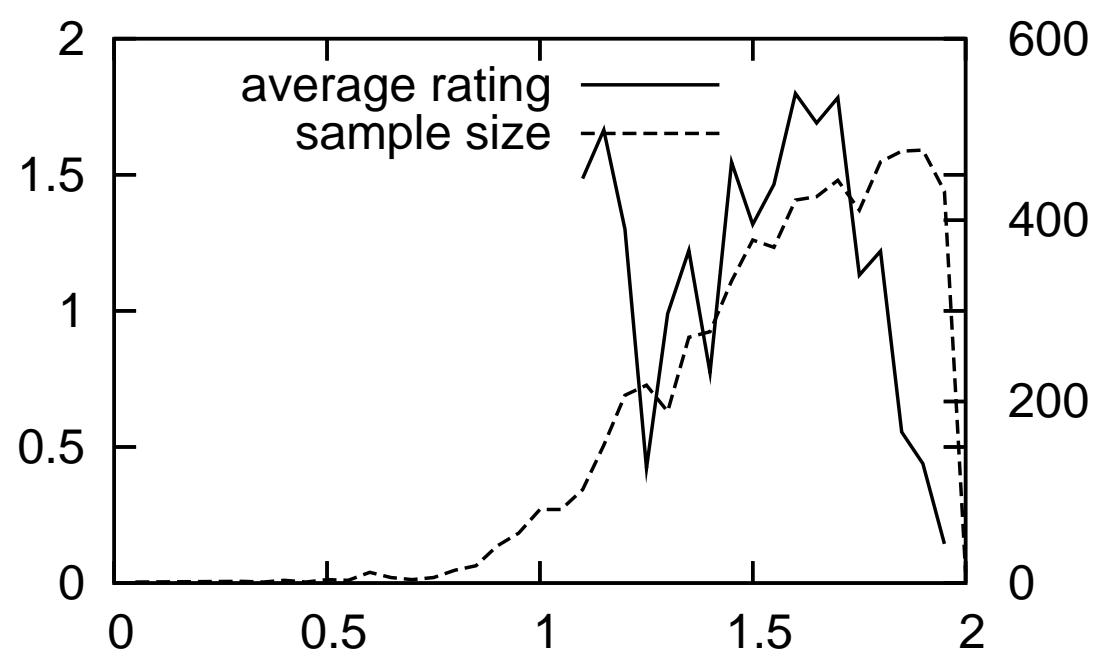

Figure 6: 